\title{
ON THE NUMBER OF SINGULARITIES OF AN ANALYTIC CURVE
}

BY

\author{
HENRY B. LAUFER( $\left.{ }^{1}\right)$
}

I. Introduction. Burau [1] and Zariski [7] showed that algebroid singularities can be classified by the knot type obtained by looking at that part of the curve lying on the boundary of a small polydisc (or ball) centered at the singularity. In this paper, by examining the isotopy class of the link obtained by looking at that part of an analytic curve lying on the boundary of an unbounded polydisc, we will obtain information about the genus and the number of singularities of that part of the curve lying within the polydisc. Thus, we get global instead of local resuits.

As an application to algebraic geometry, we give a topological proof of a Plücker formula. Also, for many proper maps of Riemann surfaces into $C^{2}$, we can count the number of points where the map fails to be regular or one-one.

The complex analysis and the knot theory are in different sections, with independent proofs.

II. Counting singularities. Let $S$ be a one-dimensional analytic subvariety in a neighborhood of $B_{R}=\left\{\left(z_{1}, z_{2}\right) \in C^{2}|| z_{1} \mid \leqq R\right\}$ such that $S \cap B_{R}$ is compact. More generally, since we are not concerned with $S$ outside of $B_{R}, S$ can be the image of a continuous map $F=\left(f_{1}, f_{2}\right): M \rightarrow B_{R}$, where $M$ is a compact complex 1-manifold with boundary, $F(\partial M) \subset \partial B_{R}, F$ is holomorphic on the interior of $M$, and $F$ is one-one on $\partial M$, the topological boundary of $M$. Topologically $\partial B_{R}$ is the product of a 1-sphere $S^{1}$ and an open 2-cell $E^{2}$. We shall coordinatize $\partial B_{R}$ by $z_{2}$ and the argument of $z_{1} . F$ restricted to $\partial M$ gives a link $L_{R}: \partial M \rightarrow \partial B_{R}$. We shall use $L_{R}$ to refer to either the map $L_{R}$ or to its image. We want to count the singularities of $F$ using topological properties of $L_{R}$.

All links in this paper will have a finite number of components.

To see what $L_{R}$ looks like, we approximate $L_{R}$ by $L_{R-\varepsilon}$, which is $F$ restricted to $F^{-1}\left(\partial B_{R-\varepsilon}\right)=\partial M_{R-\varepsilon}$. Choose $\varepsilon$ so that $f_{1}^{\prime}(z) \neq 0$ on $\partial M_{R-\varepsilon}$. As we traverse a component of $\partial M_{R-\varepsilon}$ in the positive direction, $\arg z_{1}$ in the image is strictly monotone increasing since $f_{1}$ is orientation preserving and a local homeomorphism. $H_{1}\left(\partial B_{R-\varepsilon} ; Z\right) \approx Z$ and we choose a generator which has an increasing $z_{1}$ argument. Then the homology class of $L_{R-\varepsilon}$ is, by the argument principle, equal in number to the number of zeroes of $f_{1}$. Since as $\varepsilon \rightarrow 0, L_{R-\varepsilon} \rightarrow L_{R}$, for small enough $\varepsilon$ (see

Received by the editors December 4, 1967.

( $\left.{ }^{1}\right)$ Research sponsored by the Air Force Office of Scientific Research, Office of Aerospace Research, under Contract No. F44620-67-6-0029. 
Lemma 2 for the case of arbitrary links) the links $L_{R-\varepsilon}$ are all isotopic via homeomorphisms which have strictly monotone increasing $z_{1}$ arguments.

Definition 1. Let $L=\left(f_{1}, f_{2}\right): \bigcup S_{i}^{1} \rightarrow \partial B_{R}$ be a link with a monotone increasing $z_{1}$ argument. Approximate $L$ by differentiable links $\bar{L}_{\varepsilon}$ having positive $\bar{f}_{1}$ derivatives. The self-winding $\operatorname{sw}(L)$ of $L$ is

$$
\lim _{\varepsilon \rightarrow 0} \operatorname{sw}\left(\bar{L}_{\varepsilon}\right)=\lim _{\varepsilon \rightarrow 0} \frac{1}{2 \pi} \int_{\cup S_{i}^{1}} \sum_{z^{\prime}} d\left(\arg \left(\bar{f}_{2}\left(z^{\prime}\right)-\bar{f}_{2}(z)\right),\right.
$$

where $\bar{f}_{1}$ and $\bar{f}_{2}$ are the coordinates for $\bar{L}_{\varepsilon}$ and where, for fixed $z$, the sum is over all $z^{\prime}$ such that $\bar{f}_{1}\left(z^{\prime}\right)=\bar{f}_{1}(z)$ and $z^{\prime} \neq z$.

Thus sw $(L)$ looks at the change in argument between points having the same $z_{1}$ coordinate and is integer valued. Since sw $\left(\bar{L}_{\varepsilon}\right)$ depends continuously on $\bar{L}_{\varepsilon}$ and is integer valued, sw $(L)$ is well defined and is unchanged if we deform $L$ through links with monotone increasing $z_{1}$ argument. In the next section, we shall see that sw $(L)$ is an isotopy invariant that can be defined for any link in $S^{1} \times E^{2}$.

Definition 2. Let $L$ and $L^{\prime}$ be links in $\partial B_{R}$ with monotone increasing $z_{1}$ arguments. Approximate $L$ and $L^{\prime}$ by differentiable links $\bar{L}_{\varepsilon}$ and $\bar{L}_{\varepsilon}^{\prime}$ with positive $\bar{f}_{1}$ derivatives.

$$
l\left(L, L^{\prime}\right)=\lim _{\varepsilon \rightarrow 0} l\left(\bar{L}_{\varepsilon}, \bar{L}_{\varepsilon}^{\prime}\right)=\lim _{\varepsilon \rightarrow 0} \frac{1}{2 \pi} \int \sum_{z^{\prime}} d\left(\arg \bar{f}_{2}\left(z^{\prime}\right)-\bar{f}_{2}(z)\right),
$$

where $\bar{f}_{1}$ and $\bar{f}_{2}$ are the coordinates and where, for fixed $z \in \bar{L}_{\varepsilon}$, the sum is over $z^{\prime}$ such that $\bar{f}_{1}\left(z^{\prime}\right)=\bar{f}_{1}(z)$ and $z^{\prime} \in \bar{L}_{\varepsilon}^{\prime}$. The integration is over $z$ in $\bar{L}_{\varepsilon}$.

$l\left(L, L^{\prime}\right)$ is just the usual linking number if we embed $S^{1} \times E^{2}$ in $S^{3}$ in the usual manner. In our case, this corresponds to replacing $\partial B_{R}$ by

$$
S^{3}=\left\{\left(z_{1}, z_{2}\right)|\sup | z_{1}|,| z_{2} \mid / N=R\right\}
$$

where $N$ is chosen sufficiently large so that $L$ and $L^{\prime}$ lie in $S^{3}$.

We must assign multiplicities to the singularities of $F$. Nodes (i.e. points in the image of $F$ where the image is locally two intersecting submanifolds with different tangent planes) receive multiplicity one. Any singularity can be approximated by some fixed positive number of nodes (see Corollary 2), which is its multiplicity.

Statement (4) below is a weaker result than one of Martinelli [4], who shows that $B_{R}$ can be quite arbitrary.

THEOREM 1. Let $F=\left(f_{1}, f_{2}\right): M \rightarrow B_{R}$ be a continuous map from $M$, a compact Riemann surface with boundary to $B_{R}=\left\{\left(z_{1}, z_{2}\right) \in C^{2}|| z_{1} \mid \leqq R\right\}$ such that $F(\partial M)$ $\subset \partial B_{R}, F$ is holomorphic on the interior of $M$, and $F$ is one-one on $\partial M . F$ restricted to $\partial M$ defines a link $L$. Then

$$
\operatorname{sw}(L)=(h-1)+(\mu-1)+2 g+2 d,
$$

where $h$ is the homology class of $L, \mu$ is the number of components of $L, g$ is the genus of $M$, and $d$ is the number of singularities of $F$. 
If $F_{1}$ and $F_{2}$ are two such maps, then

(4) $l\left(L_{1}, L_{2}\right)$ is the number of intersections of $F\left(M_{1}\right)$ with $F\left(M_{2}\right)$.

Proof. We can first approximate $F$ arbitrarily closely on the interior of $M$ so that the only singularities of the map are nodes and so that $f_{1}^{\prime}(z)$ has only simple zeroes. $L_{R-\varepsilon}$ is deformed only slightly so that $\mathrm{sw}\left(L_{R-\varepsilon}\right)$ is unchanged. Singularities are replaced by the appropriate number of nodes. Thus we may assume that the only singularities of $F$ are nodes, where we must replace $B_{R}$ by $B_{R-\varepsilon}$. There can be no singularities near $\partial M$ since as $\varepsilon \rightarrow 0$, sw $\left(L_{R-\varepsilon}\right)$ is constant and thus $d$ on the right side of (3) cannot increase.

On $M$, form the function $p(z)=\prod_{z^{\prime}} f_{2}\left(z^{\prime}\right)-f_{2}(z)$ where $z^{\prime} \neq z$ and $f_{1}\left(z^{\prime}\right)=f_{1}(z)$. $p(z)$ is holomorphic except possibly at a $z$ such that $f_{1}^{\prime}\left(z^{\prime}\right)=0$ for some $z^{\prime}$ with $f_{1}\left(z^{\prime}\right)=f_{1}(z)$. Since $p(z)$ is locally bounded, it must have a removable singularity at such a $z$.

$2 \pi$ sw $(L)$ is nothing more than the total change in argument for $p(z)$ as $z$ traverses $\partial M$. Thus sw $(L)$ equals the number of zeroes of $p(z) \cdot p(z)=0$ can occur only when $f_{2}(z)=f_{2}\left(z^{\prime}\right), f_{1}(z)=f_{1}\left(z^{\prime}\right), z \neq z^{\prime}$, i.e. at a node, or when $z^{\prime} \rightarrow z$, i.e. where $f_{1}^{\prime}(z)=0$. Every node yields two zeroes, one for each of the points which are not separated. For the other possibility, $f_{1}^{\prime}(z)=0$ is a simple zero and $f_{2}^{\prime}(z) \neq 0$ by our approximation assumption. If $f_{1}(z)$ is thus locally $z^{2}$, then $z^{\prime}=-z$ and $p(z)$ has a simple zero.

Look at $F^{-1}\left(\partial B_{r}\right)=\left\{z \in M|| f_{1}(z) \mid=r\right\}$. When $r=0$ we get $h$ points and for small $r, h$ circles (we may assume that $f_{1}(z)$ and $f_{1}^{\prime}(z)$ have disjoint zeroes). Let $r$ increase to $R-\varepsilon$. The number of components of $F^{-1}\left(\partial B_{r}\right)$ increases or decreases by one each time we go through a $z$ such that $f_{1}^{\prime}(z)=0$. The number of components cannot change at other $z$ since they can only combine or divide when $f_{1}$ is not a local homeomorphism, can only, by the minimum principle, appear at points where $f_{1}(z)=0$, and, by the maximum principle, cannot contract to points and disappear. We start with $h$ components, end with $\mu$ components and have $g$ handles. Since $M$ is connected, there must be $(h-1)+2 g+(\mu-1)$ places where $f_{1}^{\prime}(z)=0$ $((h-1)$ points to join the $h$ components, 2 points for each of the handles, and $(\mu-1)$ points to end with $\mu$ components, although generally not in that order). Hence (3) is proved:

(4) is easier to prove. Let, for $z \in M_{1}, g(z)=\prod_{z^{\prime}} f_{2}\left(z^{\prime}\right)-f_{2}(z)$ where $z^{\prime} \in M_{2}$ and $f_{1}(z)=f_{1}\left(z^{\prime}\right)$. Now argue as before.

If we have a singularity at the origin having one branch and $z_{2}=0$ gives the tangent line, then the knot corresponding to the singularity necessarily lies in $\partial B_{\varepsilon}$, for small $\varepsilon$. We shall see in the next section that generalizations of sw $(\cdot)$ serve to classify such knots in $S^{1} \times E^{2}$.

CoROllary 2. Let $K \subset \partial B_{\varepsilon}$ be the knot associated to a singularity with one branch. Then $2 d=\mathrm{sw}(K)-(h-1)$ where $d$ is the multiplicity of the singularity and $h$ is the homology class of the knot. 
Proof. Locally the singularity is given by $F=\left(z^{h}, f_{2}\right): D \rightarrow B_{\varepsilon}$, where $D$ is a disc. There is one component to the knot and $D$ is simply connected.

COROllaRy 3 (PlüCKER). Let $C$ be an irreducible algebraic curve of degree $m$ and genus $g$ having only cusps and nodes as singularities. Then

$$
g=\frac{1}{2}(m-1)(m-2)-s-n,
$$

where $s$ is the number of cusps and $n$ is the number of nodes.

Proof. Since a cusp may be parametrized by $\left(z^{2}, z^{3}\right)$ and has as corresponding knot a torus knot of type $(2,3)$, the multiplicity of a cusp is one.

Choose the line $l$ at infinity so that $C \cap l$ is $m$ distinct points, having homogeneous coordinates $\left(1, p_{i}, 0\right), 1 \leqq i \leqq m$. Then as $R=\left|z_{1}\right| \rightarrow \infty$, looking at $\partial B_{R} \cap C, z_{2} / z_{1} \rightarrow p_{i}$ and we get $m$ distinct circles, each with homology class one and each pair having linking number one. There are $m(m-1)$ ordered pairs of circles, so by (3)

$$
m(m-1)=(m-1)+(m-1)+2 g+2 n+2 s .
$$

We can get some information about trying to embed Riemann surfaces to $\boldsymbol{C}^{2}$. See [3, pp. 220-221] for the construction of proper maps of the needed type.

Corollary 4. Let $F=\left(f_{1}, f_{2}\right): S \rightarrow C^{2}$ be a proper holomorphic map of $S$, an open Riemann surface of finite genus $g$ with $\mu$ boundary components, such that $f_{1}$ is an almost proper map. Let $L_{R}$ be links corresponding to components $M_{R}$ of

$$
\left\{z \in S|| f_{1}(z) \mid \leqq R\right\}
$$

such that $M_{R} \subset M_{R+1}$ and $\bigcup M_{R}=S$. Then $F$ is an embedding if and only if for all sufficiently large $R$

$$
\mathrm{sw}\left(L_{R}\right)=\left(h_{R}-1\right)+(\mu-1)+2 g \text {. }
$$

Proof. The maximum principle shows that $M_{R}$ is, for sufficiently large $R$, a Riemann surface with boundary of genus $g$ and with $\mu$ boundary components. Since every singularity of $F$ has multiplicity at least one, (5) is just the condition that there be no singularities.

III. Isotopy invariance. If we deform a link $L$ in $T=S^{1} \times E^{2}$, (1) will not, in general, suffice to define sw $(L)$ since $\arg z_{1}$ need not be increasing and singularities may occur in the integral, even assuming that $L$ remains differentiable. We shall define, in the topological category, i.e. for all continuous embeddings $L: \bigcup_{i} S_{i}^{1} \rightarrow T$, an isotopy invariant $\mathrm{sw}(L)$ which coincides with (1) when $L$ has an increasing $z_{1}$ argument. $L$, or more precisely the image of $L$, need not be connected, although stronger invariants may be obtained by looking at each component of $L$ separately. We retain our parametrization of

$$
T=B_{1}=\left\{\left(z_{1}, z_{2}\right) \in C^{2}|| z_{1} \mid=1\right\} .
$$


Let $\left\{L_{i}\right\}$ be the components of $L$. Let $h_{i}$ be the homology class of $L_{i}$. To every point $\left(z_{1}, z_{2}\right)$ of $L_{i}$ assign $\arg z_{1}$ which, after starting somewhere and varying the argument continuously, is well defined modulo $2 \pi h_{i}$. To generalize sw $(L)$ from (1), start at $p \in L$ and $p^{\prime} \in L$ such that $\arg p \equiv \arg p^{\prime}, \bmod 2 \pi$ and $p$ and $p^{\prime}$ are in different components or $\arg p \not \equiv \arg p^{\prime}, \bmod 2 \pi h_{i}$. Look at the argument of the $z_{2}$ coordinate of $p^{\prime}-p$ and follow the change in argument as $p$ varies around $L$. When the derivative of $\arg p^{\prime}$ changes sign we reverse the direction in which we are traversing $L$. Eventually we return to our starting pair. sw $(L)$ is the sum of all changes in the $z_{2}$ argument which occur in this manner. Thus,

Definition 3. Let $L$ be a polygonal link, $L=\left(f_{1}, f_{2}\right): \bigcup_{i} S_{i}^{1} \rightarrow T$ such that $f_{1}$ is not constant on any segment of $L$. Let $h_{i}$ be the homology class of $L_{i}$, a component of $L$. Modulo $2 \pi h_{i}$, assign continuously $z_{1}$ arguments to the points of $L_{i}$ for all $i$. Let $s$ be arclength on $\bigcup_{i} S_{i}^{1}$. Then

$$
\operatorname{sw}(L)=\frac{1}{2 \pi} \int_{\cup_{i} s_{i}^{1}} \sum_{s^{\prime}} \varepsilon\left(s^{\prime}\right) \frac{d\left(\arg \left(f_{2}\left(s^{\prime}\right)-f_{2}(s)\right)\right)}{d s} d s
$$

where the sum is over all $s^{\prime}$ such that $f_{1}\left(s^{\prime}\right)=f_{1}(s)$ and $s$ and $s^{\prime}$ are in different components or $\arg f_{1}\left(s^{\prime}\right) \not \equiv \arg f_{1}(s) \bmod 2 \pi h_{i} . \varepsilon\left(s^{\prime}\right)$ is the sign of the derivative of the $z_{1}$ argument at $s^{\prime}$.

sw $(L)$ is necessarily an integer, for look at the pairs $\left(s, s^{\prime}\right)$. They form a 1-complex and every 0 -cell, occurring when $s$ or $s^{\prime}$ is a vertex, is on the boundary of an even number of 1-cells, and in fact is on the boundary of two 1-cells unless both $s$ and $s^{\prime}$ are vertices and the derivatives of both $\arg \left(f_{1}(s)\right)$ and $\arg \left(f_{1}\left(s^{\prime}\right)\right)$ change sign at $s$ and $s^{\prime}$. In this last case, $\left(s, s^{\prime}\right)$ is on the boundary of four 1-cells. Thus as we follow $\arg \left(f_{2}\left(s^{\prime}\right)-f_{2}(s)\right)$ around the 1-complex, we eventually return to our starting point, without retracing paths. (6) shows that sw $(L)$ depends continuously on $f_{2}$. Hence sw $(L)$ is invariant under homotopies which keep the $z_{1}$-coordinates fixed and which have no self-intersections at $s$ and $s^{\prime}$ such that $s$ and $s^{\prime}$ are in different components or $\arg f_{1}(s) \not \equiv \arg f_{1}\left(s^{\prime}\right)$, $\bmod 2 \pi h_{i}$.

$T=S^{1} \times E^{1} \times E^{1}$. Projecting onto $S^{1} \times E^{1}$, we get a link diagram for $L$. By arbitrarily small deformations of $L$ we may get a link whose projection is in general position (see [2, p. 7]). To each double point, or crossing, $c$ we assign $\eta(c)=1$ if the overpass crosses the underpass from right to left and $\eta(c)=-1$ if the overpass crosses from left to right.

Lemma 1. Let $L$ be a polygonal link, $L=\left(f_{1}, f_{2}\right): \bigcup_{i} S_{i}^{1} \rightarrow T$ such that $f_{1}$ is not constant on any segment of $L$ and such that the link diagram of $L$ is in general position. Then

$$
\mathrm{sw}(L)=\sum \eta(c)
$$

where the sum is over those crossings whose under-and overpass are in different components of $L$ or have different arguments, modulo $2 \pi h_{i}$ where $h_{i}$ is the homology class of $L_{i}$, the component of $L$ containing the under-and overpass. 
Proof. Without changing $f_{1}$ or the link diagram we deform $L$ so that it lies in the plane of $S^{1} \times E^{1}$ except near the crossings. This does not change sw $(L)$.

$$
\frac{d\left(\arg f_{2}\left(s^{\prime}\right)-f_{2}(s)\right)}{d s}=0
$$

except near the crossings. One checks the cases, i.e. $\eta= \pm 1$ and the under- and overpass have increasing or decreasing $z_{1}$ arguments and one sees that (7) is correct.

In $T$, measure distance in $S^{1}$ by arclength in $S^{1}$.

Lemma 2. Let $L$ and $\bar{L}$ be polygonal links

$$
L=\left(f_{1}, f_{2}\right): \bigcup_{i} S_{i}^{1} \rightarrow T, \quad \bar{L}=\left(\bar{f}_{1}, \bar{f}_{2}\right): \bigcup_{i} S_{i}^{1} \rightarrow T
$$

such that $f_{1}$ and $\bar{f}_{1}$ are not constant on any segment and such that the link diagrams of $L$ and $\bar{L}$ are in general position. Let $\delta>0$ be such that

$$
\left|\arg f_{1}\left(s^{\prime}\right)-\arg f_{1}(s)\right|<\frac{2 \pi}{9} \quad \text { if }\left|s-s^{\prime}\right|<\delta .
$$

Let

$$
m=\inf \left[\max \left|f_{1}\left(s^{\prime}\right)-f_{1}(s)\right|,\left|f_{2}\left(s^{\prime}\right)-f_{2}(s)\right|\right]
$$

with inf taken over $\left\{s, s^{\prime}|| s-s^{\prime} \mid \geqq \delta\right.$ or $s$ and $s^{\prime}$ are in different components of $\left.L\right\}$.

Let $\bar{L}$ satisfy the following conditions:

(i) The preimages $\left\{v_{j}\right\}$ of the vertices of $\bar{L}$ coincide with the preimages of the vertices of $L$.

(ii) Let $\Delta$ be a triangle with vertices

$$
\left\{L\left(v_{j}\right), \bar{L}\left(v_{j}\right), L\left(v_{j+1}\right)\right\} \quad \text { or } \quad\left\{L\left(v_{j+1}\right), \bar{L}\left(v_{j}\right), \bar{L}\left(v_{j+1}\right)\right\}
$$

and let $\Delta^{\prime}$ be another such triangle. Then $\Delta$ and its interior intersect the sides of $\Delta^{\prime}$ only in isolated points and sides in common.

(iii) In their link diagrams, the vertices of $L$ and $\bar{L}$ are all distinct.

(iv) $\left|f_{1}(s)-\bar{f}_{1}(s)\right|<\min (2 \pi / 9, m / 6)$ and $\left|f_{2}(s)-\bar{f}_{2}(s)\right|<m / 6$ for all $s$.

Then sw $(L)=\mathrm{sw}(\bar{L})$.

Proof. $m>0$ by compactness.

We shall use the triangles $\Delta$ to construct a suitable homotopy between $L$ and $\bar{L}$ and then look at the changes in the link diagram. We may add vertices to $L$ and $\bar{L}$ so that $\left|v_{j}-v_{j+1}\right|<\delta^{\prime}$, with $\delta^{\prime}$ such that $\delta^{\prime} \leqq \delta$ and $\left|f_{1}\left(s^{\prime}\right)-f_{1}(s)\right|<m / 6$ and $\left|f_{2}\left(s^{\prime}\right)-f_{2}(s)\right|<m / 6$ if $\left|s^{\prime}-s\right|<\delta^{\prime}$, and not change the hypotheses of the lemma.

Replace the segment with end points $L\left(v_{j}\right)$ and $L\left(v_{j+1}\right)$ by two segments, $\left(L\left(v_{j}\right), \bar{L}\left(v_{j}\right)\right)$ and $\left(\bar{L}\left(v_{j}\right), L\left(v_{j+1}\right)\right)$. Then replace $\left(\bar{L}\left(v_{j}\right), L\left(v_{j+1}\right)\right)$ by $\left(L\left(v_{j+1}\right), \bar{L}\left(v_{j+1}\right)\right)$ and $\left(\bar{L}\left(v_{j}\right), \bar{L}\left(v_{j+1}\right)\right)$. This moved $\left(L\left(v_{j}\right), L\left(v_{j+1}\right)\right)$ to $\left(\bar{L}\left(v_{j}\right), \bar{L}\left(v_{j+1}\right)\right)$. Move $\left(L\left(v_{j+1}\right), L\left(v_{j+2}\right)\right)$ to $\left(\bar{L}\left(v_{j+1}\right), \bar{L}\left(v_{j+2}\right)\right)$ in a similar fashion (replacing also $\left(L\left(v_{j+1}\right), \bar{L}\left(v_{j+1}\right)\right)$ by $\left(L\left(v_{j+2}\right), \bar{L}\left(v_{j+2}\right)\right)$. Eventually we get a homotopy $H$ (requiring a small change of the parametrization of $\bar{L}), H: \cup S_{i}^{1} \times I \rightarrow T$. 
Use $H$ to assign a continuously varying argument, modulo $2 \pi h_{i}$, on each component in the image of $H$. The replacement of segments as defined in the previous paragraph is just like $[6$, p. 8$]$ except that there may be some intersections. Since (iii) prevents pathologies, an intersection corresponds to changing an underpass to an overpass. We wish first to show that at any such crossing the $z_{1}$ arguments of the under- and overpasses, which must be in the same component, are equal. Hence sw $(L)$, by Lemma 1 , will be unchanged.

Suppose $f_{1}(s, t)=f_{1}\left(s^{\prime}, t\right)$ and $f_{2}(s, t)=f_{2}\left(s^{\prime}, t\right)$.

$$
\left|\arg f_{1}(s, t)-\arg f_{1}(s)\right|<\frac{2 \pi}{3} \quad \text { and } \quad\left|\arg f_{1}\left(s^{\prime}, t\right)-\arg f_{1}\left(s^{\prime}\right)\right|<\frac{2 \pi}{3}
$$

from considering the possible variation from $\arg f_{1}\left(v_{j}\right)$ or $\arg f_{1}\left(v_{j+1}\right)$ in $\Delta$. Similarly,

$$
\begin{array}{ll}
\left|f_{1}(s, t)-f_{1}(s)\right|<m / 2, & \left|f_{1}\left(s^{\prime}, t\right)-f_{1}\left(s^{\prime}\right)\right|<m / 2, \\
\left|f_{2}(s, t)-f_{2}(s)\right|<m / 2, & \left|f_{2}\left(s^{\prime}, t\right)-f_{2}\left(s^{\prime}\right)\right|<m / 2 .
\end{array}
$$

$\left|s-s^{\prime}\right| \geqq \delta$ or $s$ and $s^{\prime}$ in different components is impossible for then either

$$
\left|f_{1}\left(s^{\prime}\right)-f_{1}(s)\right| \geqq m \text { or }\left|f_{2}\left(s^{\prime}\right)-f_{2}(s)\right| \geqq m \text {. }
$$

If $\left|s-s^{\prime}\right|<\delta$, then $\left|\arg f_{1}\left(s^{\prime}\right)-\arg f_{1}(s)\right|<2 \pi / 9$ and indeed for all $s^{\prime}$ such that $\left|s-s^{\prime}\right|<\delta$. From

$$
\left|\arg f_{1}(s, t)-\arg f_{1}(s)\right|<2 \pi / 3 \text { and }\left|\arg f_{1}\left(s^{\prime}, t\right)-\arg f_{1}\left(s^{\prime}\right)\right|<2 \pi / 3,
$$

which hold for all $t$, we see that $\left|\arg f_{1}\left(s^{\prime}, t\right)-\arg f_{1}(s, t)\right|<2 \pi$ and hence $\arg f_{1}\left(s^{\prime}, t\right)$ $=\arg f_{1}(s)$ as desired.

Thus the intersections in $H$ have no effect on sw $\left(L_{t}\right)$. Otherwise $H$ is an isotopy and we need only observe the effect of the operations $\Omega_{1}, \Omega_{2}$ and $\Omega_{3}[6$, pp. 7-8] on sw $\left(L_{t}\right)$, calculated from Lemma 1 . But $\Omega_{1}, \Omega_{2}$ and $\Omega_{3}$ do indeed leave sw $(L)$ invariant.

If $L$ is any continuous link and we approximate $L$ by polygonal links $L^{\prime}$ and $L^{\prime \prime}$ such that $f_{1}$ is not constant on any segment and the link diagrams of $L^{\prime}$ and $L^{\prime \prime}$ are in general position, Lemma 2 indicates how to construct $\bar{L}$ such that sw $\left(L^{\prime}\right)=\operatorname{sw}(\bar{L})$ $=\mathrm{sw}\left(L^{\prime \prime}\right)$, if $L^{\prime}$ and $L^{\prime \prime}$ are sufficiently close to $L$.

Definition 4. Let $L: \bigcup_{i} S_{i}^{1} \rightarrow T$ be a link. sw $(L)=\operatorname{sw}(\bar{L})$ where $\bar{L}$ is any polygonal link such that $\bar{f}_{1}$ is not constant on any segment of $\bar{L}$, the link diagram of $\bar{L}$ is in general position, and $\bar{L}$ is sufficiently close to $L$.

THEOREM 5. Let $H=\left(f_{1}, f_{2}\right):\left(\bigcup_{i} S_{i}^{1}\right) \times I \rightarrow T$ be an isotopy between the links $L_{0}=\left(f_{1}, f_{2}, 0\right)$ and $L_{1}=\left(f_{1}, f_{2}, 1\right)$. Then sw $\left(L_{0}\right)=\mathrm{sw}\left(L_{1}\right)$.

Proof. Use $H$ to assign continuously $z_{1}$ arguments to the image of $H$. Since $\left(\bigcup S_{i}^{1}\right) \times I$ is compact, $m(t)=m\left(f_{1}, f_{2}, t\right)$ of Lemma 2 is bounded away from 0 . Thus we may use Lemma 2 to find polygonal links $\bar{L}_{t}$ which approximate $\left(f_{1}, f_{2}, t\right)$ and which have the same sw $\left(\bar{L}_{t}\right)$ for all $t$. Thus sw $\left(L_{0}\right)=\operatorname{sw}\left(L_{1}\right)$. 
Besides sw $(L)$, we may define isotopy invariants $\mathrm{sw}_{i}(L)$ as follows. For simplicity, we shall assume that $L$ is connected, $i$ is an integer.

Definition 5. Let $K: S^{1} \rightarrow T$ be a knot. Approximate $K$ by polygonal knots $\bar{K}_{\varepsilon}$ such that $\bar{f}_{1}$ is not constant on any segment of $\bar{K}_{\varepsilon}$. Let $h$ be the homology class of $K$. For $i \not \equiv 0 \bmod h$, let

$$
\operatorname{sw}_{i}\left(\bar{K}_{\varepsilon}\right)=\frac{1}{2 \pi} \int_{s^{1}} \sum_{s^{\prime}} \varepsilon\left(s^{\prime}\right) \frac{d\left(\arg \left(f_{2}\left(s^{\prime}\right)-f_{2}(s)\right)\right)}{d s} d s
$$

where the sum is over all $s^{\prime}$ such that $\arg f_{1}\left(s^{\prime}\right)-\arg f_{1}(s) \equiv 2 \pi i, \bmod 2 \pi h$.

$$
\mathrm{sw}_{i}(K)=\lim _{\varepsilon \rightarrow 0} \operatorname{sw}_{i}\left(\bar{K}_{\varepsilon}\right) \text {. }
$$

THEOREM 6. Let $K: S^{1} \rightarrow T$ be a knot. $\mathrm{sw}_{i}(K)$ is independent of the choice of the approximating knots and is an isotopy invariant. If $h$ is the homology class of $K$,

$$
\mathrm{sw}(K)=\sum_{i=1}^{h-1} \mathrm{sw}_{i}(K) \text {. }
$$

Theorem 6 is proved, using Lemma 2, in the same manner as Theorem 5 was proved.

If an algebroid singularity has a Puiseaux expansion [5, p. 163-164]

$$
y=a_{1} x^{n_{1} / m_{1}}+a_{2} x^{n_{2} / m_{1} m_{2}}+a_{3} x^{n_{3} / m_{1} m_{2} m_{3}}+\cdots
$$

with $m_{1} \leqq n_{1}, n_{1} m_{2}<n_{2}, n_{2} m_{3}<n_{3}, \ldots$ and $\left(m_{1}, n_{1}\right)=1,\left(m_{2}, n_{2}\right)=1,\left(m_{3}, n_{3}\right)=1, \ldots$ then the associated knot is a tubular knot $A_{k}$ given by the symbol

$$
\left\{\left(m_{1}, n_{1}\right),\left(m_{2}, n_{2}\right), \ldots,\left(m_{k}, n_{k}\right)\right\} \text {. }
$$

Without changing the singularity or the knot, we may assume that $m_{i} \neq 1,1 \leqq i \leqq k$. $m=m_{1} \cdots m_{k}$ is the homology class of $A_{k}$. $A_{k}$ is constructed inductively as follows. Let $A_{0}=\{(1,1)\}$ be the circle $z_{2}=0$, oriented positively. $A_{k}$ winds $m_{k}$ times about $A_{k-1}$ in the direction of the parallels and $n_{k}$ times about $A_{k-1}$ in the direction of the meridians, where $A_{k}$ must lie on a small tubular surface centered at $A_{k-1}$.

THEOREM 7. Let $K$ be the knot in $\partial B_{\varepsilon}$ associated to a singularity at the origin with one branch given by (8). Then the $m_{j}$ and the $n_{j}$ are determined by the homology class of $K$ and the $\mathrm{sw}_{i}(K)$.

Proof. We shall show by induction that

(9)

$$
\begin{aligned}
& \mathrm{sw}_{i}=m_{k} \cdots m_{2} n_{1} \quad \text { if } i \not \equiv 0 \quad \bmod m_{1} \\
& \mathrm{sW}_{i}=m_{k} \cdots m_{3} n_{2} \quad \text { if } i \equiv 0 \quad \bmod m_{1}, i \not \equiv 0 \quad \bmod m_{1} m_{2} \\
& \mathrm{sw}_{i}=m_{k} \cdots m_{j+1} n_{j} \quad \text { if } i \equiv 0 \quad \bmod m_{1} \cdots m_{j-1}, i \not \equiv 0 \quad \bmod m_{1} \cdots m_{j} \\
& \mathrm{sw}_{i}=n_{k} \quad \text { if } i \equiv 0 \quad \bmod m_{1} \cdots m_{k-1} \text {. }
\end{aligned}
$$


Since $n_{i+1}>n_{i} m_{i+1}$, the $\mathrm{sw}_{i}$ are listed in (9) with strictly increasing values, so that the $m_{j}$ are all determined, using $m=m_{1} \cdots m_{k}$ to determine $m_{k}$ from the previous $m_{j}$ and $m$, the homology class of $K$. Then the $n_{j}$ are also all determined.

To prove (9), when $k=1$, we have a torus knot of type $\left(m_{1}, n_{1}\right)$ and (9) is true. We now proceed by induction.

In general $A_{k}$ winds $m_{k}$ times about $A_{k-1}$ in the direction of the parallels. Thus if $i \equiv 0 \bmod m_{1} \cdots m_{j-1}$ and $i \not \equiv 0 \bmod m_{1} \cdots m_{j}$ and we look at $s^{\prime}$ and $s$ with $\arg s^{\prime}-\arg s \equiv 2 \pi i, \bmod 2 \pi m$, after $s$ winds about $S^{1} m_{1} \cdots m_{k-1}$ times, we see that $f_{2}\left(s^{\prime}\right)-f_{2}(s)$ changes argument by $m_{k-1} \cdots m_{j+1} n_{j} 2 \pi$. But instead of completing a full circuit around $A_{k}$, we have only gone around $A_{k-1}$ and $A_{k}$ winds $m_{k}$ times around $A_{k-1}$ in this direction. Thus $\mathrm{sw}_{i}=m_{k} m_{k-1} \cdots m_{j+1} n_{j}$.

Finally if $i \equiv 0 \bmod m_{1} \cdots m_{k-1}$, we have that $A_{k}$ winds $n_{k}$ times around $A_{k-1}$ in the direction of the meridians, so that $\mathrm{sw}_{i}(K)$ is the same as for the $\operatorname{knot}\left\{\left(m_{k}, n_{k}\right)\right\}$ or just $n_{k}$, as desired.

\section{REFERENCES}

1. W. Burau, Kennzeichnung der Schlauchknoten, Abh. Math. Sem. Univ. Hamburg 9 (1932), 125-133.

2. R. H. Crowell and R. H. Fox, Introduction to knot theory, Ginn, New York, 1963.

3. R. C. Gunning and H. Rossi, Analytic functions of several complex variables, PrenticeHall, Englewood Cliffs, N. J., 1965.

4. E. Martinelli, Sulle intersezioni delle curve analitiche complesse, Rend. Mat. e Appl. (5) 14 (1955), 422-430.

5. J. E. Reeve, A summary of results in the topological classification of plane algebroid singularities, Univ. e Politec. Torino Rend. Sem. Mat. 14 (1955), 159-187.

6. K. Reidemeister, Knotentheorie, Springer, Berlin, 1932.

7. O. Zariski, On the topology of algebroid singularities, Amer. J. Math. 54 (1932), 453-465.

Massachusetts Institute of Technology, CAMBridge, MasSACHUSETTS 